\title{
PURIFICAÇÃo DE ÓLEO BÁSICO DE MAMONA PARA FORMULAÇÃO DE BIOLUBRIFICANTES UTILIZANDO DESTILAÇÃ̃O MOLECULAR
}

\author{
J. A. C. da SILVA ${ }^{1}$, A. H. S. de OLIVEIRA ${ }^{2}$ e A. C. R. CAETANO 3 \\ ${ }^{1}$ Centro de Pesquisa e Desenvolvimento da Petrobras \\ ${ }^{2}$ Centro de Pesquisa e Desenvolvimento da Petrobras \\ ${ }^{3}$ Universidade de Fortaleza, Centro de Ciências e Tecnologia \\ E-mail para contato: jose.andre@petrobras.com.br
}

\begin{abstract}
RESUMO - O desenvolvimento de biolubrificantes tem sido estimulado no meio acadêmico e na indústria com o objetivo de reduzir os danos causados por vazamento de óleos lubrificantes minerais em equipamentos e motores. Biolubrificantes derivados de óleos vegetais apresentam alto valor agregado devido a sua biodegradabilidade e por serem de fontes renováveis e abundantes na natureza. Contudo, a sua produção ainda apresenta desvantagens como a dificuldade na separação e purificação do éster e coprodutos. Este trabalho apresenta uma alternativa como solução para esse problema através da aplicação da metodologia de purificação por destilação molecular de um óleo básico produzido a partir da transesterificação do biodiesel de mamona com trimetilolpropano. A partir de testes realizados em uma unidade piloto de destilação molecular, foi possível a obtenção de um óleo básico purificado, caracterizado como biolubrificante, com rendimento de $70 \%(\mathrm{~m} / \mathrm{m})$ utilizando uma temperatura de evaporação a $240{ }^{\circ} \mathrm{C}$ e de condensação a $30^{\circ} \mathrm{C}$ sob pressão de $0,001 \mathrm{mmHg}$, com as seguintes propriedades físico-químicas: índice de viscosidade superior a 101, ponto de fluidez de $-36{ }^{\circ} \mathrm{C}$ e acidez total inferior a $0,5 \mathrm{mgKOH} / \mathrm{g}$.
\end{abstract}

\section{INTRODUÇÃO}

O lubrificante oriundo do petróleo é um produto de alto valor agregado e com grande utilidade no setor industrial, principalmente em motores e equipamentos. Contudo, trata-se de um material resistente à biodegradação e com grande potencial de poluição do meio ambiente através do descarte inadequado. Devido às novas políticas de preservação do meio ambiente associadaa às reservas limitadas de petróleo, e consequentemente aos seus derivados, o desenvolvimento e aprimoramento de bioprodutos estão sendo estimulados no meio acadêmico e industrial.

Biolubrificantes derivados de óleo vegetais apresentam propriedades físico-químicas semelhantes aos lubrificantes de origem mineral, além de proporcionar vantagens no que diz respeito ao meio ambiente, como biodegradabilidade e por serem oriundos de fontes renováveis. Algumas rotas reacionais já foram desenvolvidas e estabelecidas para a produção de biolubrificantes a partir de ésteres oriundos de oleaginosas. 
Alguns fatores influenciam a escolha da oleaginosa, tais como: disponibilidade, logística e o efeito da mesma nas propriedades físico-químicas do biolubrificante. Uma oleaginosa interessante é a mamona, devido à mesma ser produzida em várias regiões do Brasil e de fácil cultivo. Além disso, o óleo produzido a partir dessa oleaginosa não compete com a indústria alimentícia. Outra vantagem é que o óleo de mamona possui propriedades desejáveis para produção de biolubrificante, como uma melhor densidade, viscosidade, solubilidade em álcool e lubricidade, quando comparado a outros óleos de origem vegetal [Silva, 2011].

Esse trabalho apresenta a produção de biolubrificante a partir de biodiesel de mamona com trimetilolpropano (TMP) utilizando catalisador a base de estanho. O produto da reação é nomeado como óleo básico não purificado, por conter coprodutos e ainda não está especificado de acordo com as propriedades requeridas para a aplicabilidade de um biolubrificante. As etapas convencionais de purificação do óleo básico normalmente são dispendiosas, com alto gasto energético e formação de efluentes. A destilação molecular apresenta-se como uma alternativa na purificação de biolubrificantes por ser um processo que ocorre dentro de uma ampla faixa de temperatura e sob alto vácuo, favorecendo a separação e purificação de produtos com alto peso molecular e/ou termicamente sensíveis e minimizando efeitos de decomposição térmica, além de não requerer o uso de solventes que possam comprometer a pureza e as propriedades físicoquímicas do produto desejado [Batistella e Maciel, 1996].

Essa tecnologia é um caso particular de evaporação, por se tratar de um processo em que não ocorre equilíbrio líquido-vapor, pois as superfícies de evaporação e de condensação estão a uma distância mínima necessária para que as moléculas evaporadas alcancem facilmente a superfície do condensador, antes de ocorrer colisão entre elas, sem nenhuma resistência. Deste modo, a destilação molecular proporciona a separação/purificação de produtos sob condições menos severas que as tecnologias alternativas e no menor tempo possível [Wrang et al., 2009]. Entretanto, esse processo demanda um estudo minucioso na determinação das variáveis operacionais, visto que pequenas alterações nas condições de processo comprometem o rendimento e as propriedades físico-químicas dos produtos purificados.

Este trabalho estuda as variáveis operacionais do processo de destilação molecular, como temperatura, pressão e vazão, com o objetivo de fracionar éster e coprodutos, visando o aprimoramento das propriedades físico-químicas do óleo básico para a sua aplicação como biolubrificante.

\section{METODOLOGIA}

\subsection{Processamento}

O óleo básico foi produzido em uma unidade piloto de produção de biolubrificantes, utilizando Biodiesel de Mamona e Trimetilolpropano (TMP) como reagentes. A reação de transesterificação ocorreu na presença de um catalisador a base de estanho, apresentando uma conversão entre 65 e $75 \%$. 
A purificação do óleo básico foi realizada na unidade piloto de destilação molecular de filme descendente com operação contínua, constituída por módulos equivalentes: um evaporador (área: $0,06 \mathrm{~m}^{2}$ ), um condensador (área: $0,10 \mathrm{~m}^{2}$ ) e um sistema de alto vácuo, nomeados de Evaporador (EV) e Destilador Molecular (DM). A única diferença entre os módulos é representada por uma bomba difusora no DM que permite um vácuo de 0,001 $\mathrm{mmHg}$. A Figura 1 apresenta um diagrama de blocos das etapas de purificação.

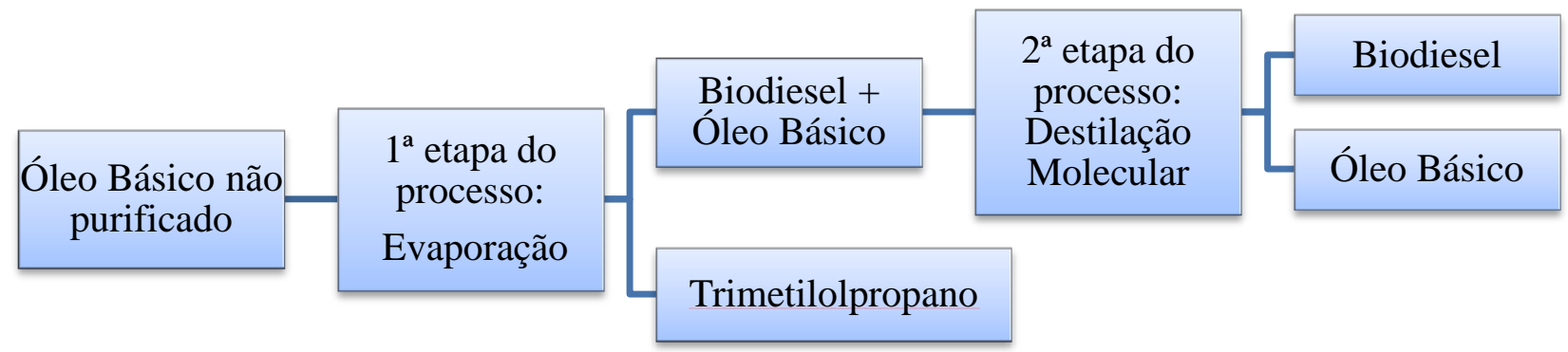

Figura 1 - Fluxograma do processo de produção.

Neste estudo, o processo aplicado durante os testes de purificação do óleo básico apresentou dois níveis de pressão, os quais estão descritos abaixo:

Evaporação (short path distillation): Realizada na $1^{\mathrm{a}}$ etapa do processo no Evaporador (EV), na qual a distância do evaporador para o condensador permite a livre transferência das moléculas evaporadas, sem obstruções. O nível de vácuo está entre $1 \mathrm{mmHg}$ e $0,1 \mathrm{mmHg}$ e apresenta um mínimo equilíbrio entre o líquido e o vapor.

Destilação molecular: Realizada na $2^{\mathrm{a}}$ etapa do processo no Destilador Molecular (DM), a distância entre o evaporador e o condensador é a mesma do EV, no entanto, a faixa de vácuo está entre 0,1 a $0,001 \mathrm{mmHg}$, o que permite a eliminação de barreiras de gás na superfície de evaporação e não apresenta equilíbrio líquido-vapor.

As duas etapas foram primordiais para obtenção de um produto com propriedades físicoquímicas adequadas e livre de contaminantes (TMP e Biodiesel). A primeira etapa realizada no EV tem como objetivo a remoção completa do TMP contido no produto reacional (óleo básico não purificado), evitando obstruções nas linhas de processo devido ao seu alto ponto de fusão $\left(58^{\circ} \mathrm{C}\right)$.

O efeito da temperatura de evaporação (Tev) na remoção do TMP foi avaliado a partir dos resultados de cromatografia gasosa, observando o rendimento desse componente na corrente de concentrado (carga para $2^{\mathrm{a}}$ etapa). A temperatura de condensação foi definida a $70{ }^{\circ} \mathrm{C}$ a partir do ponto fusão do TMP $\left(58^{\circ} \mathrm{C}\right)$ e do ponto de ebulição $\left(79,1^{\circ} \mathrm{C}\right)$ na pressão de $0,17 \mathrm{mmHg}$, estimado pelo nomógrafo desenvolvido pela Sigma-Aldrich. A vazão de carga nessa etapa foi $1 \mathrm{~kg} / \mathrm{h}$ devido à literatura reportar que quanto menor a vazão de carga maior será a eficiência na separação dos produtos, por proporcionar um maior tempo de residência [Fregolente et al., 2007]. 
A segunda etapa foi realizada no DM para separar o biodiesel do óleo básico. A faixa de temperatura estudada foi de 200 a $250{ }^{\circ} \mathrm{C}$. Inicialmente realizou-se um teste a $170{ }^{\circ} \mathrm{C}$ baseando-se no ponto de ebulição estimado em $161,4{ }^{\circ} \mathrm{C}$ a $0,01 \mathrm{mmHg}$, entretanto a separação não foi suficiente para promover um óleo básico com viscosidade e ponto de fluidez desejado. Novos testes foram realizados alterando a Tev de $10 \mathrm{em} 10{ }^{\circ} \mathrm{C}$, quando se observou que essa faixa de temperatura seria apropriada ao escopo desse trabalho. A pressão foi estabelecida em torno de $0,001 \mathrm{mmHg}$ e a temperatura de condensação aproximadamente $30^{\circ} \mathrm{C}$.

\subsection{Métodos Analíticos}

Para a obtenção da viscosidade cinemática e índice de viscosidade dos produtos analisados, foi utilizado um viscosímetro Stabinger, Anton Paar Modelo SVM 3000, no qual a viscosidade é obtida através do movimento à velocidade constante de um tubo que comporta a mistura. O ponto de fluidez foi determinado através do ICL Modelo CPP5GS, de acordo com a ASTM D97. O índice de acidez total (IAT), que indica a degradação oxidativa do óleo, foi obtido de acordo com a ASTM D664 utilizando o Titrando 905 da Metrohm.

\section{RESULTADOS E DISCUSSÕES}

Inicialmente, foram analisadas algumas propriedades físico-químicas do biodiesel de mamona e do óleo básico produzido a partir de uma reação de transesterificação utilizando catalisador a base de estanho. A partir da Tabela 1 pode-se verificar a diferença na viscosidade e no índice de viscosidade (IV) do produto em relação ao seu reagente.

A viscosidade é uma propriedade fundamental quando se refere a lubrificantes, definida como a resistência do fluido ao escoamento devido à formação de um filme no meio envolvente, ou seja, um bom lubrificante deve apresentar uma viscosidade adequada que proporcione a proteção de superfícies metálicas dos equipamentos e motores. Em relação ao ponto de fluidez (PF), o mesmo é definido como a temperatura mais baixa em que o material ainda escoa. É uma propriedade de extrema importância, quando os lubrificantes são aplicados em ambientes de baixa temperatura, podendo danificar peças e equipamentos, caso ocorra alguma obstrução relacionada à solidificação dos mesmos ou até mesmo à formação de pequenos cristais.

Nesse caso, o PF não se alterou significativamente, pelo fato do Biodiesel de Mamona já possuir um PF relativamente baixo. Entretanto, o teor de TMP no óleo básico exerce um efeito desfavorável, uma vez que o mesmo possui alto ponto de fusão $\left(58^{\circ} \mathrm{C}\right)$.

Tabela 1 - Propriedades físico-químicas da matéria-prima

\begin{tabular}{|c|c|c|r|c|}
\hline & $\mu \mathrm{a} 40{ }^{\circ} \mathrm{C}(\mathrm{cSt})$ & $\mu \mathrm{a} 100{ }^{\circ} \mathrm{C}(\mathrm{cSt})$ & $\mathrm{IV}$ & $\mathrm{PF}\left({ }^{\circ} \mathrm{C}\right)$ \\
\hline Biodiesel de Mamona & 16,5 & 3,3 & 51 & -27 \\
\hline Óleo básico não purificado & 161,9 & 15,3 & 95 & -30 \\
\hline
\end{tabular}

Apesar do óleo básico não purificado apresentar um IV próximo de 95 e PF em torno de - 
$30{ }^{\circ} \mathrm{C}$, ainda não poderá ser enquadrado como um biolubrificante, uma vez que a presença dos contaminantes (Biodiesel e TMP) prejudica outras propriedades, como, por exemplo, a estabilidade oxidativa. $\mathrm{O}$ uso de aditivos melhora esta propriedade, porém contribui para o aumento do custo total na produção do biolubrificante. Por outro lado, a destilação molecular pode ser utilizada para purificar o óleo básico, consequentemente melhorando essas e outras propriedades.

Na primeira etapa de purificação, foi realizado o estudo do efeito da Tev na remoção do TMP, demonstrado na Figura 2. A faixa de temperatura testada foi de 120 a $180{ }^{\circ} \mathrm{C}$, mantendo-se constantes todas as outras variáveis operacionais. O teor (rendimento) de TMP diminui na corrente de concentrado (biodiesel + óleo básico) com o aumento da Tev devido ao aumento da evaporação de voláteis. Desta forma, uma alta Tev promove uma eficiente separação. De acordo com a Figura 2, a remoção de TMP foi completa em temperaturas acima de $160^{\circ} \mathrm{C}$.

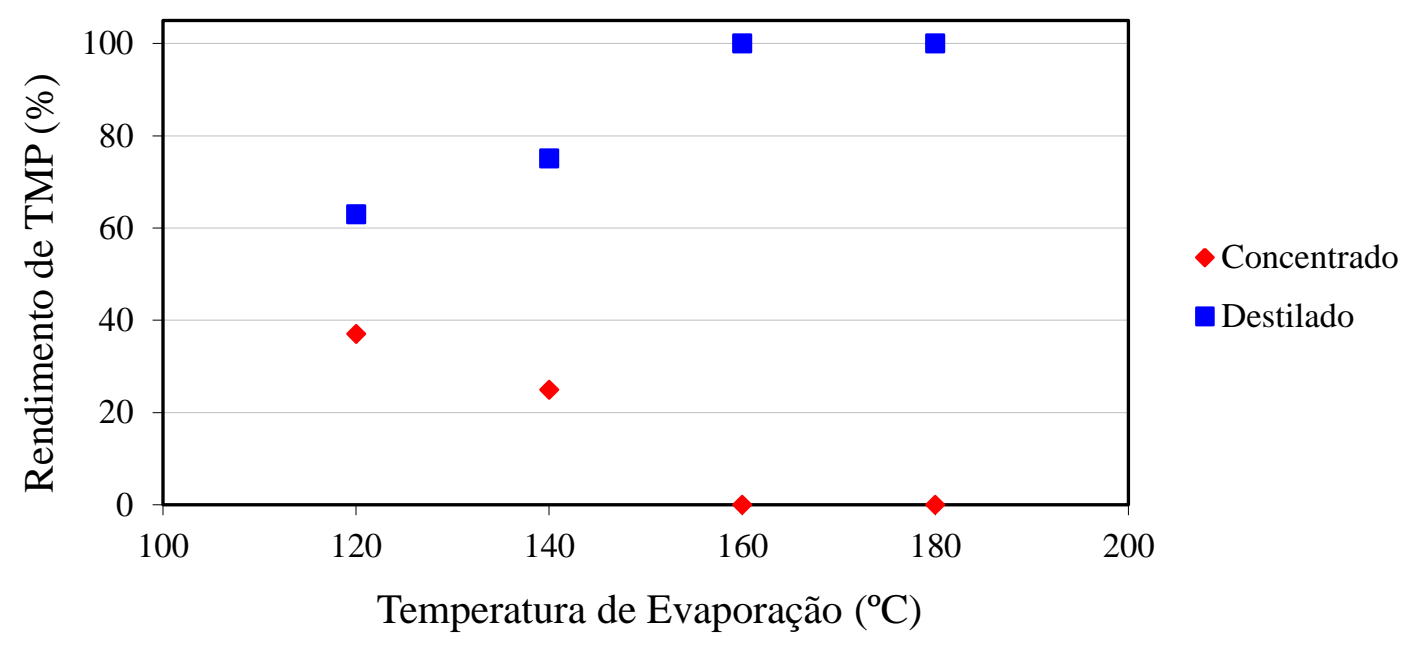

Figura 2 - Efeito da temperatura de evaporação no rendimento de TMP nas correntes de destilado e concentrado. Testes realizados nas seguintes condições: temperatura de condensação $70{ }^{\circ} \mathrm{C}$, temperatura da carga $80^{\circ} \mathrm{C}$, vazão de carga $1 \mathrm{~kg} / \mathrm{h}$, rotação das palhetas $840 \mathrm{RPM}$.

A partir dos resultados de cromatografia das amostras do destilado, observou-se que os componentes leves do Biodiesel também eram vaporizados, portanto o produto utilizado como carga para a DM (concentrado da EV) recebia uma mistura de Biodiesel (livre de leves) + Óleo Básico, permitindo um fechamento adequado do balanço material, já que a perda de material volátil para o sistema de vácuo no DM foi minimizada pela remoção dos mesmos na etapa anterior.

O efeito da Tev no DM, etapa de remoção de Biodiesel, nas propriedades do produto final também foi avaliado. De acordo com as Figuras 3 e 4, a temperatura influencia positivamente a viscosidade do óleo básico, assim como o IV, tornando o produto adequado para aplicações como lubrificante. Contudo, o rendimento mássico diminui com o aumento da Tev devido à evaporação 
dos componentes de menor peso molecular, podendo-se alcançar um ponto ótimo na temperatura de $240{ }^{\circ} \mathrm{C}$.

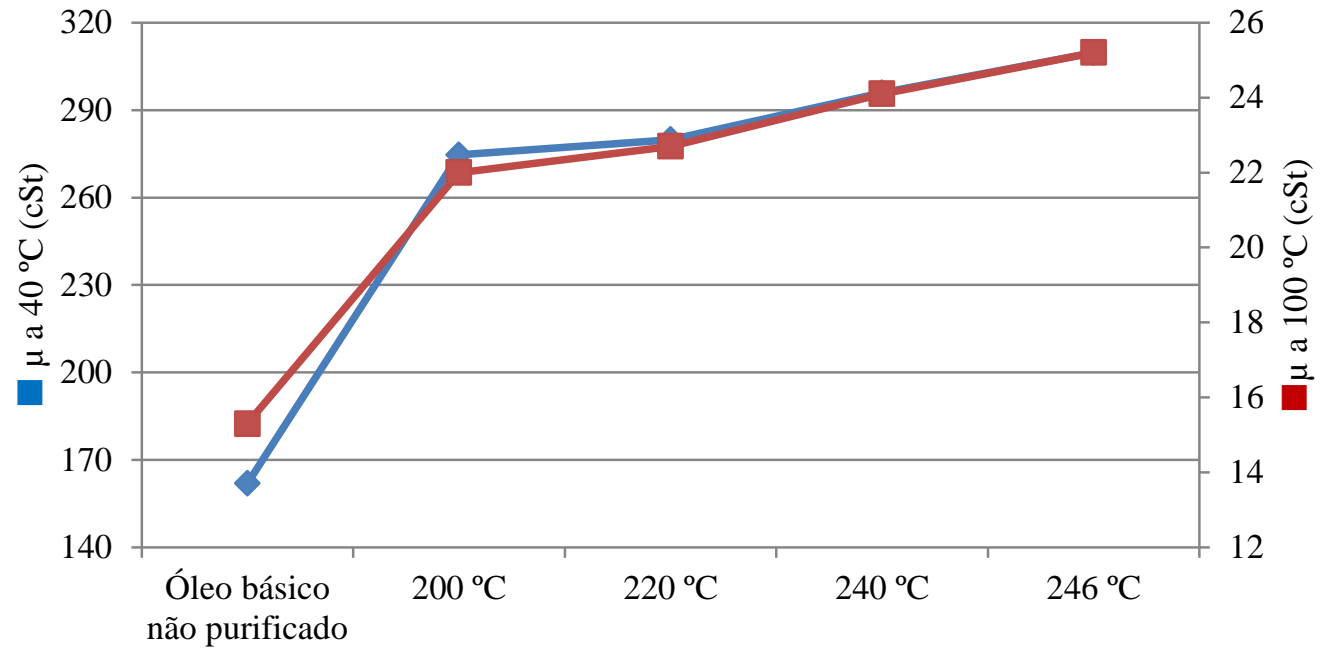

Temperatura de evaporação

Figura 3 - Efeito da temperatura de evaporação na viscosidade do óleo básico. Testes realizados nas seguintes condições: temperatura de condensação $30^{\circ} \mathrm{C}$, temperatura da carga $80^{\circ} \mathrm{C}$, rotação das palhetas $840 \mathrm{RPM}$ e pressão de $0,001 \mathrm{mmHg}$.

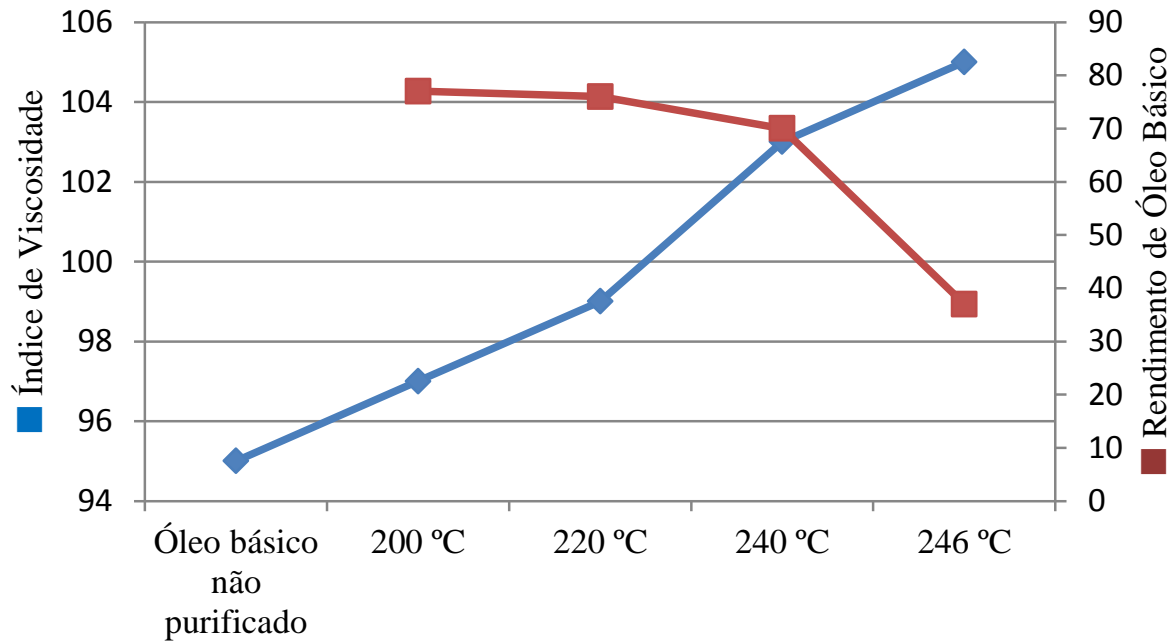

Temperatura de Evaporação

Figura 4 - Efeito da temperatura de evaporação no IV do óleo básico e rendimento mássico no processo de destilação molecular. Testes realizados nas seguintes condições: temperatura de condensação $30^{\circ} \mathrm{C}$, temperatura da carga $80^{\circ} \mathrm{C}$, rotação das palhetas $840 \mathrm{RPM}$ e pressão de 0,001 $\mathrm{mmHg}$. 
De acordo com a Figura 5, pode-se observar que a faixa de temperatura estudada não variou o PF (ponto de fluidez) do produto, porém o mesmo não foi observado em relação ao índice de acidez total (IAT). Na temperatura de $200{ }^{\circ} \mathrm{C}$, o óleo purificado apresentou acidez elevada possivelmente devido ao teor de biodiesel que não foi destilado, assim como na temperatura de $246^{\circ} \mathrm{C}$, sendo esta justificada pela oxidação do óleo.

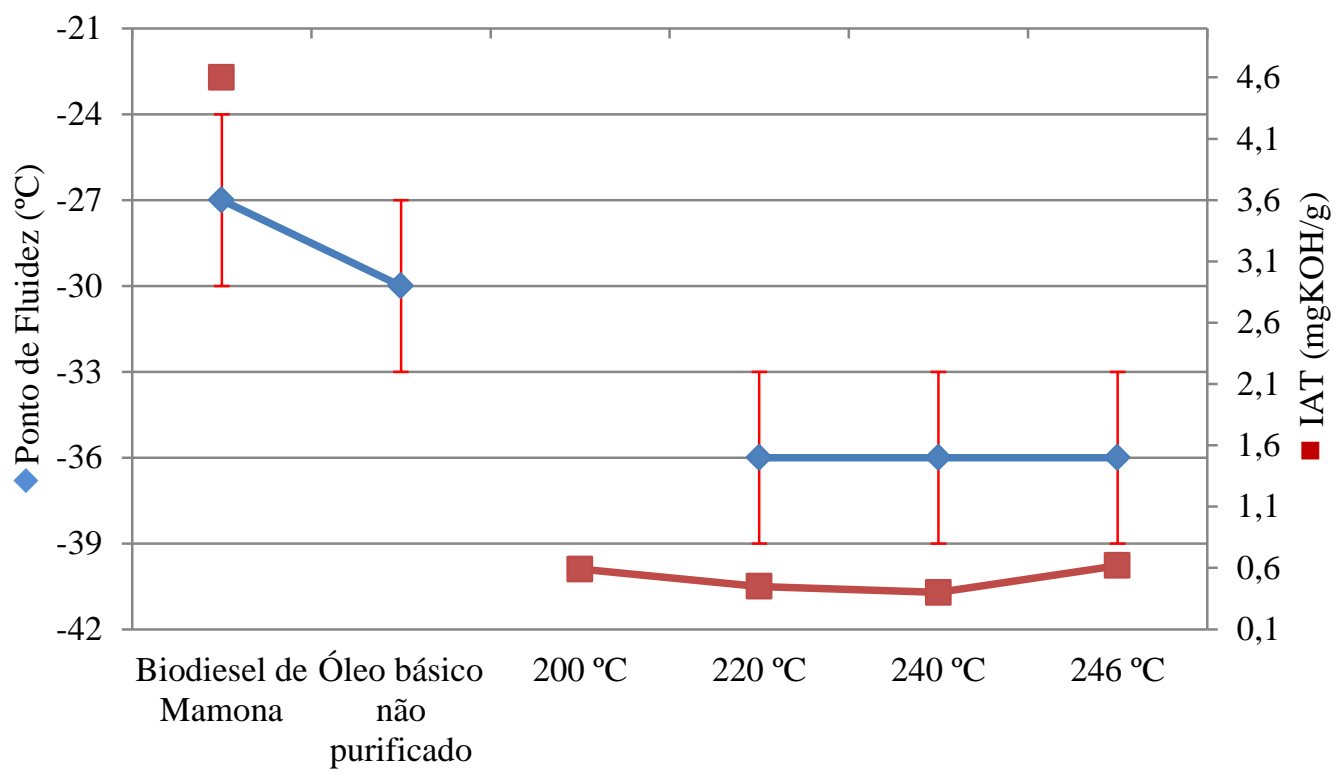

Temperatura de Evaporação

Figura 5 - Efeito da temperatura de evaporação no PF e IAT do óleo básico. Testes realizados nas seguintes condições: temperatura de condensação $30^{\circ} \mathrm{C}$, temperatura da carga $80{ }^{\circ} \mathrm{C}$, rotação das palhetas 840 RPM e pressão de 0,001 mmHg. Obs: Barra de erro do PF definida em $+-3^{\circ} \mathrm{C}$.

Métodos convencionais de purificação de óleo básico vegetal comparados ao uso da destilação molecular foram estudados por Perez, 2009. Segundo o autor, a lavagem ácida e a neutralização "in situ" do meio reacional com $\mathrm{H}_{2} \mathrm{SO}_{4}$ apresentam bons rendimentos, entretanto comprometem o produto de interesse, como o aumento do índice de acidez devido à utilização de solventes, mostrando que a destilação molecular é mais vantajosa.

\section{CONSIDERAÇÕES FINAIS}

O presente trabalho mostrou que a destilação molecular pode ser utilizada na purificação de óleo básico oriundo de Biodiesel de Mamona, aprimorando as suas propriedades físico-químicas e adequando o mesmo à aplicabilidade como óleo lubrificante para, por exemplo, o setor marítimo.

O óleo básico foi produzido em diversas condições operacionais, contudo a condição que melhor enquadrou as propriedades físico-químicas avaliadas foi: vazão de carga $1 \mathrm{~kg} / \mathrm{h}(\mathrm{EV})$, pressão de $0,001 \mathrm{mmHg}(\mathrm{DM})$ e temperatura de evaporação $240{ }^{\circ} \mathrm{C}(\mathrm{DM}) . \mathrm{O}$ óleo básico purificado apresentou rendimento de $70 \%$, com as seguintes propriedades físico-químicas: 
viscosidade de 295,9 e 24,10 a 40 e $100{ }^{\circ} \mathrm{C}$ respectivamente, IV igual a 103 , PF a $-36^{\circ} \mathrm{C}$ e IAT de $0,4 \mathrm{mgKOH} / \mathrm{g}$.

\section{REFERÊNCIAS}

SILVA, J. A. C. Biodegradable Lubricants and Their Production Via Chemical Catalysis. Tribology - Lubricants and Lubrification, ISBN 978-953-307-371-2, 2011.

WRANG, S.; GU Y.; LIU Q.; YAO Y.; GUO Z.; LUO Z.; CEN K. Separation of bio-oil by molecular distillation. Fuel Processing Technology, v. 90, p. 738-745, 2009.

BATISTELLA, C. B.; MACIEL, M. R. W. Modeling, simulation and analysis of molecular distillators: centrifugal and falling film. Computer Chemical Engineering, v. 20, p. 819-824, 1996.

FREGOlENTE, L. V.; FREGOLENTE, P. B. L.; CHICUTA, A. M.; BATISTELlA, C. B.; MACIEL, M. R. W.; MACIEL FILHO, R. Effect of operating conditions on the concentration of monoglycerides using molecular distillation. Chemical Engineering Research and Design, p. 1524-1528, 2007.

PEREZ, H. I. Q. Produção de biolubrificantes para usos especiais. Tese de Mestrado. 2009. 